\title{
Capabilities of Lock-in Thermography for Non-destructive Inspection of Fibre Reinforced Composites
}

by T. Ullmann, Y. Shi and R. Aoki

German Aerospace Center, Institute of Structures and Design, Pfaffenwaldring 38-40, 70569 Stuttgart, Germany, thomas.ullmann@dlr.de, yuan.shi@dlr.de, r.aoki@dlr.de

\begin{abstract}
Fundamental knowledge of process parameters for the manufacturing of fibre reinforced composites is essential for design and engineering of high performance lightweight structures. This concerns oxide and non-oxide fibre reinforced ceramics (CMC) as well as reinforced polymers (e.g. CFRP). The manufacturing of a layered fibre reinforced composite structure is similar to a primary shaping process and demands an efficient and consistently process monitoring to ensure sufficient quality standards. Consequently, more detailed non-destructive investigations focusing the composite's microstructure combined with the acquired knowledge of critical manufacturing process parameters are necessary. Optically excited lock-in thermography is a qualified and proven inspection technique that provides valuable information of the composite material's microstructure. For more sophisticated signal interpretation, the lock-in thermography measurement results are compared with other non-destructive evaluation (NDE) techniques as reference.
\end{abstract}

\section{Introduction}

The increasing use of fibre reinforced materials and structures in aerospace industry demands intensified efforts to expand the knowledge of composite material properties as well as its load capacity and behaviour under application relevant thermal and mechanical conditions. Furthermore, enhanced and consistently process monitoring strategies are needed in order to achieve high quality standards for each manufacturing procedure. In that sense, high sophisticated non-destructive inspection techniques are indispensible tools to evaluate the material's microstructure and therefore the success of every single process step. However, there are numerous well known and proven inspection techniques available that seem to be suitable to provide valuable information for quality assurance on fibre reinforced composite materials and structures. Acoustic methods like water coupled ultrasound inspection are the most common and widely accepted test methods especially among original equipment manufacturers and suppliers in aircraft industry. Other techniques like air coupled ultrasound inspection, X-ray, computed tomography, shearography and several active tomography methods are also used for special tasks but are not standard procedures so far. Thus, there are only few mandatory aerospace inspection standards existing, mostly related to commonly used water coupled ultrasound techniques. Non-destructive testing and evaluation as integrated part of a manufacturing process chain does also mean to inspect really large components with several metres in length. Consequently, beside the well known key criteria like non-contact measurement, imaging options, maximum failure detection resolution and defect selectivity even more aspects have to be considered: e.g. the penetration depth into the component's material (wall thickness), inspection conditions with single-sided or both-sided accessibility and - probably the most significant requirement - the working time that is needed for measurement data acquisition and evaluation on wide-spread surface areas. For example commonly used ultrasound transducers are screening the component's surface line by line until the complete area is scanned. Although there are highly-developed technical solutions available, this procedure requires quite a lot of time and as soon as the component's geometry includes bevelled surfaces and curved areas with different radiuses, it becomes difficult to receive proper acoustic signals of the component's interior layered structure. Moreover, the correct reading of ultrasound mappings requires wide experience of signal interpretation as it is sometimes quite difficult to discriminate between geometric influences, characteristic material patterns and real material defects. In contrast to that, non-contact measurement techniques like X-ray based computed tomography (CT) provide a 3-dimensional insight view with extremely high resolution. But with increasing sample size the attainable image resolution is decreasing significantly whereas the measuring time is increasing. Thus, a sufficient CT-inspection of large structure components becomes more and more difficult even when large test facilities are available. Although optical test methods cannot compete with the 3dimensional imaging options and detail resolution of computed tomography, the main benefit is their ability of singlesided contactless measurement in combination with covering a relatively large surface area that can be monitored within one exposure sequence.

Considering all this, optically excited lock-in thermography (OLT) seems to be a good compromise of all these constraints as it is analyzing the time dependent response of thermal waves that are reflected by interior boundary surfaces of a layered fibre composite material. Depending to the excitation frequency of the induced thermal waves, it is possible to collect information from different material depths and to cover a measurement area of approximately up to 1 $\mathrm{m}^{2}$ within one measurement cycle. A single-sided accessibility is absolutely sufficient as long as the laminate's thickness is not exceeding 5-6 mm (for CFRP) and the measurement time as well as data acquisition and interpretation can be 
performed within the order of a few minutes. The detection and localization of material defects or significant material changes (e.g. internal boundary surfaces) is relatively easy because variations of the material's thermal diffusivity are clearly distinguishable in the visual data mapping.

\section{Quality Assurance for Fibre Reinforced Composites}

With the complex microstructure of composite materials, there is a bunch of characteristic parameters that have to be focused on: fibre volume content, porosity, cracks, delaminated regions, foreign material inclusions (e.g. forgotten tapes or foils), fibre waviness (in plane and out of plane), regions of insufficient resin infiltration and many others. However, quality assurance in terms of process monitoring is not always a search for classical defects but also for deviations from predefined tolerances like a given fibre orientation angle, gap widths between adjacent fibre rovings, interstitial spacing at cross-over points of fibre tows or the homogeneity of the fibre-matrix distribution. Furthermore inline quality assurance also comprises consistently temperature and pressure control during thermal processes (e.g. autoclave infiltration) as well as continuous monitoring of fibre layup procedures. For process integrated NDE it means that after every manufacturing step, the resulting laminate structure is analyzed by automated non-destructive inspection techniques. More precisely, each NDE inspection station is monitoring for specific deviations or defects and is comparing the test results with given tolerance parameters. For saving manufacturing costs, it is important to detect material defects as soon as possible in order to avoid further processing with faulty structure parts that in the end cannot be used at all. The principal ideas of automated and process integrated quality assurance (PIQA) of a CFRP production line were previously described in earlier publications [1-3].

\section{Non-destructive Test Methods}

\subsection{Optically Excited Lock-in Thermography}

Optically excited lock-in thermography is a non-destructive and contactless inspection technique that is based on the propagation and reflection of thermal waves. For measurement, the specimen's surface is illuminated with sinusoidal modulated external heaters (e.g. synchronized halogen lamps). As the heat is absorbed by the specimen, a thermal wave is generated at the surface which propagates through the composite material and is reflected by any kind of internal boundary surface and thus also by internal defects. For example a delaminated region is acting as a local thermal barrier within the laminate microstructure which leads to additional attenuation and phase shift of the response signal. The interference of incoming and reflected waves is generating a harmonic oscillating radiation pattern at the specimen's surface which is detected by an infrared camera over a certain period of time. Afterwards, the electronic measurement device is analyzing a sequence of infrared images as it is monitoring the time dependent temperature evolution for each pixel of the specimen's image. With Fourier analysis it is possible to extract the amplitude and phase information of the responding thermal wave (Fig. 1).

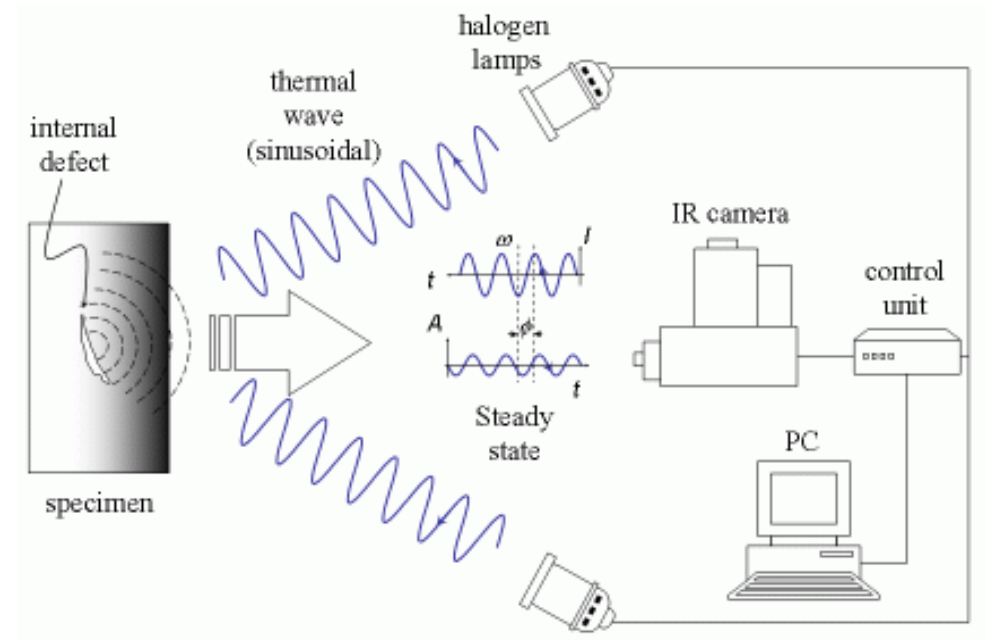

Fig. 1. Measurement principle and experimental set-up of lock-in thermography with optical excitation [4]

As the incoming thermal wave is attenuated by the material, it will only penetrate down to a certain depth which is defined as the thermal diffusion length $\mu$. Beyond this depth the amplitude would damp down below $37 \%\left(e^{-1}\right)$ of its initial value. The lower the frequency of the thermal wave the lower is the propagation velocity. For example in a polymer material the phase velocity for a $1 \mathrm{~Hz}$ wave is approximately $0.7 \mathrm{~mm} \cdot \mathrm{s}^{-1}$ while it is $0.07 \mathrm{~mm} \cdot \mathrm{s}^{-1}$ for a $0.01 \mathrm{~Hz}$ wave [6]. 
Thermal waves of lower propagation velocity (frequency) are penetrating deeper into the material. The magnitude image is given by the thermal diffusion length:

$$
\mu=\sqrt{\frac{2 \lambda}{\omega \cdot \varphi \cdot c_{p}}} \quad \Rightarrow \quad \mu=\sqrt{\frac{2 \alpha}{\omega}}=\sqrt{\frac{\alpha}{\pi \cdot f}}
$$

The angular velocity of modulation is defined as $\omega=2 \pi \cdot f\left[\mathrm{rad} \cdot \mathrm{s}^{-1}\right]$, the thermal conductivity is $\lambda\left[\mathrm{W} \cdot \mathrm{m}^{-1} \cdot \mathrm{K}^{-1}\right]$, the material density is $\rho\left[\mathrm{kg} \cdot \mathrm{m}^{-3}\right]$, the specific heat capacity is $c_{p}\left[\mathrm{~J} \cdot \mathrm{kg}^{-1} \cdot \mathrm{K}^{-1}\right]$ and $\alpha$ denotes the thermal diffusivity in perpendicular direction to the laminate structure. The signal magnitude is affected by thermal barriers (flaws) which are located in a depth less than $\mu$ whereas the signal phase still responds about twice this depth $[5,6]$. Equation (1) presumes that the heat flow is one dimensional in perpendicular direction to the fabric layers of the laminar microstructure. However, with decreasing excitation frequency and increasing penetration depth lateral heat transfer mechanisms are playing a more decisive role. Thus, the effective penetration depth is slightly decreasing compared to the calculated values and as a consequence the phase images appear more and more diffuse. Table 1 shows some calculated values of penetration depths for CFRP and different kinds of CMC material.

Table 1. Different composite materials with calculated penetration depths in parallel (II) and perpendicular $(\perp)$ fibre orientation - data from [6-11]

\begin{tabular}{|c|c|c|c|c|c|c|c|}
\hline \multicolumn{2}{|c|}{ Composite Material } & \multirow{2}{*}{$\frac{\alpha}{\left[\mathrm{cm}^{2} \cdot \mathrm{s}^{-1}\right]}$} & \multirow{2}{*}{$\frac{c_{p}}{\left[\mathrm{~J} \cdot \mathrm{g}^{-1} \cdot \mathrm{K}^{-1}\right]}$} & \multirow{2}{*}{$\begin{array}{r}\rho \\
{\left[\mathrm{g} \cdot \mathrm{cm}^{3}\right]}\end{array}$} & \multicolumn{3}{|c|}{ Thermal diffusion length $\mu$ [mm] } \\
\hline & & & & & $f=0.5 \mathrm{~Hz}$ & $f=0.05 \mathrm{~Hz}$ & $f=0.005 \mathrm{~Hz}$ \\
\hline \multirow[t]{2}{*}{ CFRP } & /I & 0.020 & \multirow[t]{2}{*}{$0.710^{2}$} & \multirow[t]{2}{*}{$1.55^{2}$} & 1.13 & 3.57 & 11.28 \\
\hline & $\perp$ & 0.004 & & & 0.50 & 1.60 & 5.05 \\
\hline \multirow[t]{2}{*}{ C/C-SiC (LSI) } & /I & $0.167^{1}$ & \multirow[t]{2}{*}{1.065} & \multirow[t]{2}{*}{2.00} & 3.26 & 10.31 & 32.61 \\
\hline & $\perp$ & $0.095^{1}$ & & & 2.46 & 7.78 & 24.59 \\
\hline \multirow[t]{2}{*}{$\mathrm{C} / \mathrm{SiC}(\mathrm{CVI})$} & /I & $0.067^{2}$ & \multirow[t]{2}{*}{$1.000^{2}$} & \multirow[t]{2}{*}{2.10} & 2.07 & 6.53 & 20.65 \\
\hline & $\perp$ & $0.033^{2}$ & & & 1.45 & 4.58 & 14.49 \\
\hline \multirow[t]{2}{*}{ WHIPOX } & /I & 0.394 & \multirow[t]{2}{*}{0.769} & \multirow[t]{2}{*}{2.72} & 5.01 & 15.84 & 50.08 \\
\hline & $\perp$ & 0.367 & & & 4.83 & 23.36 & 48.34 \\
\hline
\end{tabular}

Calibration measurements were performed with a CFRP plate that was provided with blind bore holes from the rear side. The objective was to verify the penetration depth and the accuracy of measurement for detecting small defects. As depicted in Figure 2 (schematic image on the far left), each depth class (horizontal rows) was inserted with drillings of different diameters, beginning from $8 \mathrm{~mm}$ down to $3 \mathrm{~mm}$. The calibration sample that was used here is the same one that was used by Gerhard [12] before. The OLT phase images show some of the artificial defects which are in fact blind bore holes from the rear side. Although the third row of bore holes (defect depth: $4 \mathrm{~mm}$ ) should be also visible with $0.005 \mathrm{~Hz}$, the measurement results are more or less confirming the correlation of thermal diffusion length and excitation frequency. It is obvious that with decreasing excitation frequency the penetration depth is increasing. The reason for not detecting the $4 \mathrm{~mm}$ defects with the OLT measurement at $0.005 \mathrm{~Hz}$ may be up to the specific CFRP laminate structure that could offer more resistance to a perpendicular heat flow than it is usual for this material class.

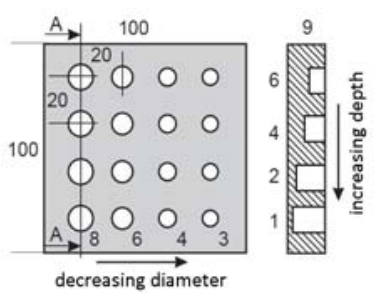

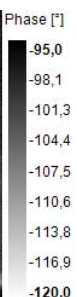

$-120,0$

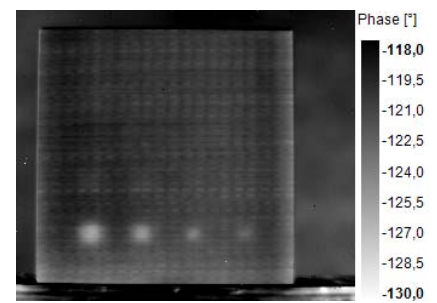

$0.01 \mathrm{~Hz}$

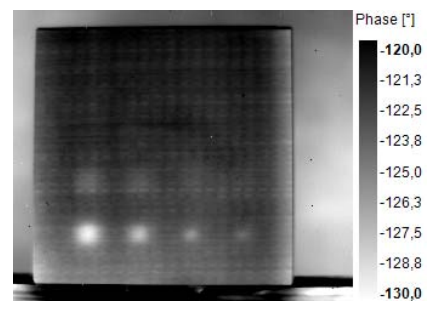

$0.005 \mathrm{~Hz}$

Fig. 2. Schematic view of a CFRP calibration sample with blind bores (left image [12]) and three OLT phase images of this sample as obtained with different excitation frequencies

\footnotetext{
1) from extrapolated measurement data [7]

2) estimated value
} 


\subsection{Contactless Ultrasound Inspection}

Air coupled ultrasound inspection was used as a reference test method in order to confirm the results of the OLT measurements. The test technique that was used here is a trough transmission mode. The emitting transducer above the test specimen is sending an ultrasound signal of approximately $120 \mathrm{kHz}$ frequency which is entering the specimen and is transmitted through the thickness of the laminate structure. As the ultrasound signal is leaving the specimen's material at the lower surface, it is detected by another transducer. For scanning the whole specimen, both transducers are moving synchronously in order to scan the component's material structure line by line. Through scan mode works on the principle that if a material defect is encountered by the transducer couple, the ultrasound signal is absorbed, reflected or simply scattered and a strongly attenuated or even no signal at all is detected by the receiver. On the corresponding ultrasound transmission image such areas appear black or at least dark blue due to the locally increased damping of the detected acoustic signal (Fig. 3). Through transmission imaging is primarily used as a confirming technique for other non-destructive test methods there is no information at which material depth the defects are located. The physical background of air coupled ultrasound measurement is described in detail by Stößel [13].

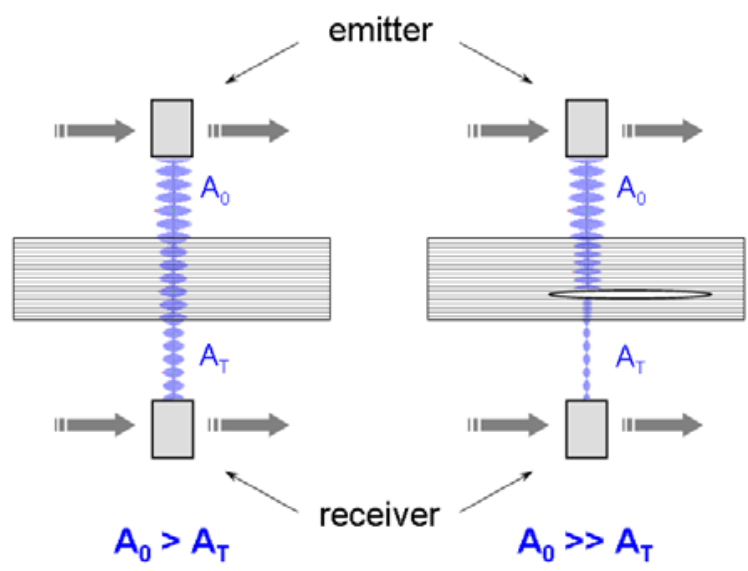

Fig. 3. Measurement principle of a through transmission mode ultrasound scan ( $A_{0}=$ amplitude of the emitted signal, $A_{T}=$ amplitude of the damped signal)

\subsection{Computed Tomography}

In a CT facility, the specimen is exposed to a cone beam of X-rays which are generated from the focal spot of an X-ray tube on the one side and are digitally monitored by a flat panel detector on the opposite side. In essence, CT is an advanced technique of radiography which allows detailed 3-dimensional views of the inner structure of a specimen or structure component. To get the full set of imaging data, the specimen is slowly rotating stepwise $(<1 \%$ step) around a single axis while the flat panel detector is monitoring hundreds of 2-dimensional X-ray projections from different orientations (Fig. 4). According to Lambert's law of absorption, equation (2) describes the attenuation of X-radiation which is passing through a homogeneous material of a certain thickness $d$.

$$
I=I_{0} \cdot e^{-\mu \cdot d} \quad \Rightarrow \quad-\ln \frac{I}{I_{0}}=\mu \cdot d
$$

However, regarding typical X-ray projections of CMC materials with a large variety of different gray values, caused by fibres, ceramic matrix and porosity, it is clear that this is no homogeneous material. Considering that the cone beam is passing a 3-dimensional microstructure which is consisting of multiple volume fractions with discrete absorption coefficients, equation 3 can be expressed as follows:

$$
I=I_{0} \cdot e^{-\int \mu(x, y, z) d s} \Rightarrow-\ln \frac{I}{I_{0}}=\int \mu(x, y, z) d s
$$

$\mu(x, y, z)$ denotes the linear absorption coefficient at each point $(x, y, z)$ within the volume of the material's inhomogeneous microstructure. The linear absorption coefficient is depending on the material's density $\rho(x, y, z)$ and the atomic number $Z(x, y, z)$ of the elements of which the material is composed at the respective location. Based on the 2-dimensional X-ray projections, the 3-dimensional CT data set is calculated by a numerical reconstruction process. Like a digital image is composed of a 2-dimensional matrix of numerous small dots (pixels), a CT scan is consisting of a 3-dimensional cloud of numerous dots that are so to say volume pixels or just "voxels". Thus, each of these voxels represent a discrete gray 
value with its accurate position within the reconstructed volume: it is one of numerous elements of the 3D-visualization of all measured $\mu(x, y, z)$ values. The attainable voxel size depends on the pixel size of the flat panel detector and the diameter $d$ of the sample's body of rotation (Fig. 4). For higher image resolution it is necessary to reduce the voxel size $V$ which is given by:

$$
V=\frac{P}{M}=\frac{P \cdot Z_{s}}{Z_{D}}
$$

Where $P$ is the detector pixel size (at DLR: $P=200 \mu \mathrm{m}$ ) and $M$ is the geometric magnification which is the ratio of the focus-detector distance $Z_{D}$ and the focus-sample distance $Z_{S}$ (Fig. 4). In general there is one rule for full volume CT scans: the smaller the specimen's rotational diameter, the higher is the attainable resolution.

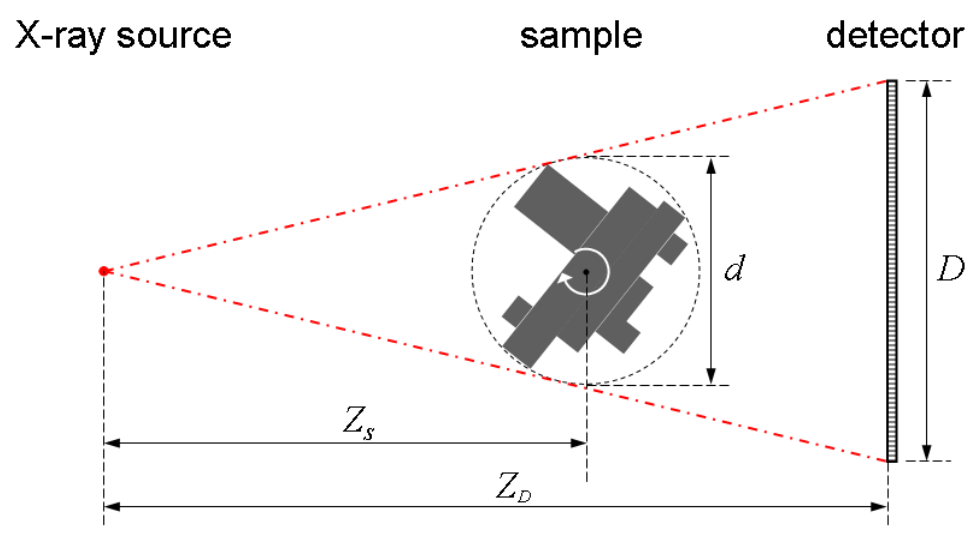

Fig. 4. Measurement configuration of a computed tomography scan

\section{Applications}

\subsection{Detection of Delaminated Areas}

$\mathrm{C} / \mathrm{SiC}$ is a carbon fibre reinforced ceramic material with $\operatorname{SiC}$ matrix $[9,10]$. The reference plate investigated here was manufactured with the chemical vapour infiltration process (CVI). The surface was sealed with a thin SiC coating and the plate's dimensions were approx. $185 \times 70 \times 5.5 \mathrm{~mm}^{3}$ (length/width/thickness). The objective of this investigation was the search and characterization of artificially induced delaminated areas by using CT. Figure 5 shows the results of the non-destructive pre-investigation with air-coupled ultrasound inspection (left image) and lock-in thermography measurements at different excitation frequencies. Some of the images of the CT inspection are shown in Figure 6 below.

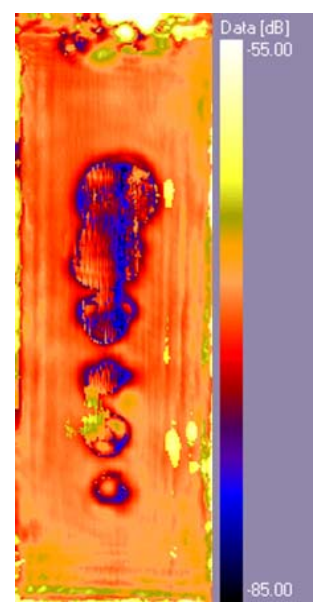

ultrasound

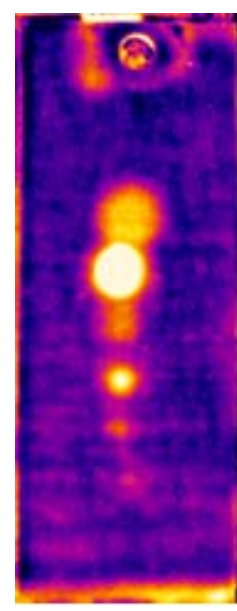

$0.3 \mathrm{~Hz}$

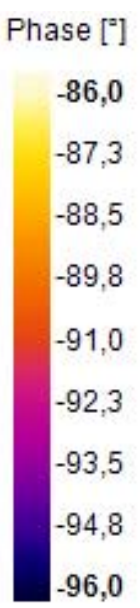

96,0

Fig. 5. Comparison of image results with air coupled ultrasound inspection and OLT analysis applied on a C/SiC (CVI) reference sample with artificially induced delaminated areas 
There are at least 6 different circular shaped delaminated regions that could have been indentified by using ultrasound and thermography inspection techniques. As already mentioned, there is no information in thickness when using air coupled ultrasound in a through transmission mode which of course affects the information value of the inspection result (Fig. 5, image on the far left). However, due to locally intensified attenuation of the air-coupled ultrasound signal $(\leq 80 \mathrm{~dB})$, the position, size and the shape of the delaminated areas in the plane of the panel is clearly visible. Not knowing the results of other investigations, it is rather difficult to say whether this is one irregular shaped delamination or an accumulation of several smaller ones. With lock-in thermography it is relatively easy to answer this question (Fig. 5, images 2, 3 and 4 from left to right): in a sense, at $0.3 \mathrm{~Hz}$, the defect signal appears with a higher resolution. Obviously, the delaminated area is an aggregation of 5 to 6 different regions that - judging from the contrast are located in different depths of the layered CMC material. This is confirmed by OLT measurements at $0.06 \mathrm{~Hz}$ and 0.01 $\mathrm{Hz}$. At lower excitation frequencies (and higher penetration depths) it becomes obvious that there are two rows, each consisting of 3 circular shaped delaminated areas of different size. In every row the diameter of these defects is decreasing from top to bottom $(\varnothing \approx 16.8 \mathrm{~mm}, 10.7 \mathrm{~mm}$ and $6.0 \mathrm{~mm})$. One row is located deeper than the other one. Although the deeper row is almost invisible in the $0.06 \mathrm{~Hz}$ phase image, which is a result of the chosen image contrast, with excitation at $0.01 \mathrm{~Hz}$ it is clear that a second, slightly displaced row of delaminated regions appears at a deeper location. The images of Figure 5 were taken by facing the back side of the C/SiC plate. The so called front side is labelled with the plate's number and is shown by the XY-slices of the CT images (Fig. 6) below. Of course, the first OLT measurements were performed by facing the plate's front side. However, after the first front side inspections at $0.3 \mathrm{~Hz}$ and $0.06 \mathrm{~Hz}$ the delaminated areas remained completely invisible. With $0.01 \mathrm{~Hz}$ one of the larger defects appeared with rather low contrast - just comparable to the upper disk-like signal on the far right image of Figure 5 (for $f=0.01 \mathrm{~Hz}$ ). In fact it is the same delamination, just viewed from the other side. The conclusion is that the location of the defects is closer to the plate's backside than to the front side surface. According to Equation (1) and considering that this CMC plate was only 5-6 mm thick, it seems rather unlikely that deeper located defects could not be found with excitation frequencies below $0.03 \mathrm{~Hz}$. Nevertheless, this was the case with OLT when facing the front side of the C/SiC plate. The reason for this may be the fact that for the calculation of penetration depths some thermal properties for CVI processed $\mathrm{C} / \mathrm{SiC}$ in Table 1 were only estimated (e.g. thermal diffusivity $\alpha$ ). Another reason may be the SiC surface coating of this plate which is another thermal barrier with different material and at least one additional boundary surface. However, even if the calculated values are not always of highest accuracy, it becomes apparent that the thermal diffusion length for CVI $\mathrm{C} / \mathrm{SiC}$ material is less than that of LSI C/C-SiC (Table 1) as was demonstrated in chapter 3.1.

For the CT inspection it was decided to choose a measurement configuration that allows displaying the $\mathrm{C} / \mathrm{SiC}$ plate with its full width $(70 \mathrm{~mm})$ on the flat panel detector. As a result, the magnification factor of the image projection was 4 and the voxel size approx. $50 \mu \mathrm{m}$. Thus, the sample could be measured in its full length within 3 different sectors which means 3 different CT measurement cycles with an adequate overlap in between. Some of the 2-dimensional CT slices are shown in Figure 6 below.

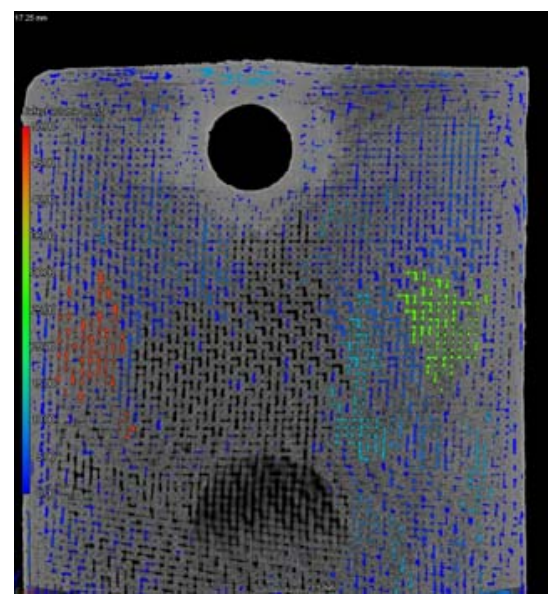

Section 1: XZ-layer \#172

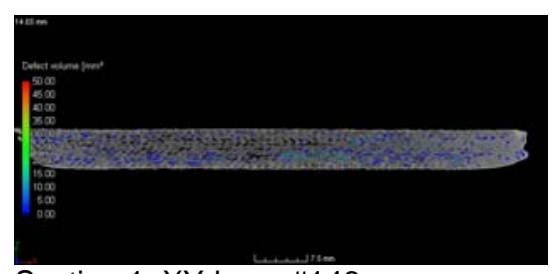

Section 1: XY-layer \#146

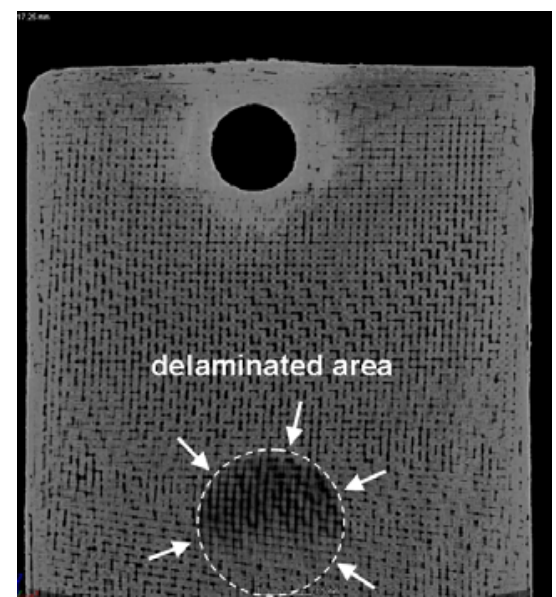

Section 1: XZ-layer \#172

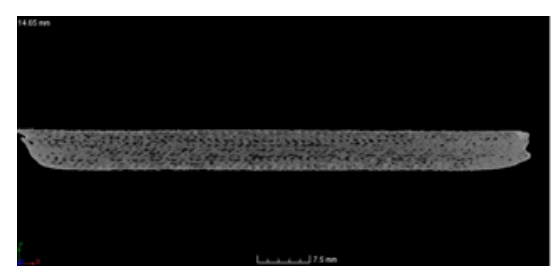

Section 1: XY-layer \#146

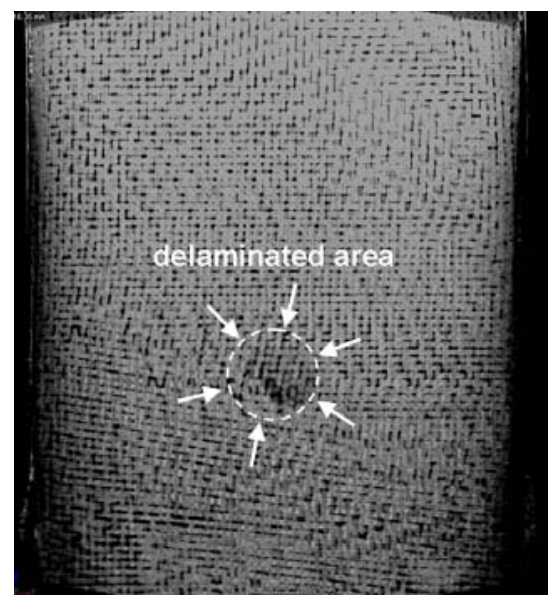

Section 3: XZ-layer \#163

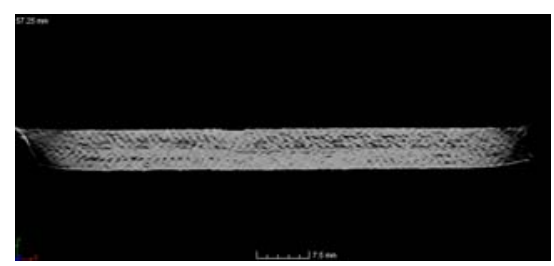

Section 3: XY-layer \#572

Fig. 6. Cross-sectional views from CT analysis of a C/SiC (CVI) reference sample with artificially induced defects 
With CT all delaminated areas were fond and clearly identified. Beside this, much more details of the composite's layered microstructure are visible on the XZ-, XY- and YZ-slice image stacks. Obviously the C/SiC material seems to be quite porous. Some of these pores were marked by using a defect finder module of the visualization software (VG Studio Max 2.1). The colours are indicating different sizes of the pores that have been identified (Fig. 6, left image). Some larger pores, outlined with bright blue, green and red colour, are also visible but these are not the kind of delaminated area that was searched for. It is assumed that these are aggregations of numerous smaller pores which are connected in a network like structure. The delaminated areas were not found by the defect search tool at all. Obviously the internal volume of this kind of flat defects was too big to be identified with the chosen settings of the automated defect search. An efficient way to find the delaminations is scrolling through the CT XZ-slices along the Y-axis and looking for darker disk-like shaped objects in the carbon fabric structure (Fig. 6, upper row).

One task was to make an attempt whether it is possible to find the delaminated areas in the cross section views (XY- \& YZ-view) or not and if so to measure the gap width in perpendicular direction to the fabric layers. However, the attempt was not successful. Even a closer look to the cross section views did not provide any informative basis for a reliable identification of delaminated areas within the porous laminate structure. In fact the resolution of the CT scans was not sufficient. According to Figure 4, it is possible to increase the resolution significantly by moving the sample towards the X-ray source and therefore increasing the magnification factor of the displayed image projection. With a microfocus CT the voxel size can be reduced down to $10 \mu \mathrm{m}$ and even less but doing so means to reduce the ascertainable sample volume as well due to the limited size of the flat panel detector. This doesn't make sense as long as there is no information about the exact location and the size of the delaminated areas in the sample. Another problem is the orientation of the laminate structure. Regarding the carbon fabric structure and the displayed shape of the found delaminated areas (Fig. 6, upper row), it becomes obvious that the plane of the XZ-slices and the orientation of the carbon fabric layers with the planar defects is slightly differing. As a consequence, the XZ-cut through the delaminated area is slightly out of plane of the circular defect's extension which makes it even more difficult to identify it - not to mention when the delamination's extension is of irregular shape.

In summary one can say that a CT inspection provides much more detailed information about a CMC's microstructure than any other non-destructive inspection technique. But in the special case of searching for planar defects like delaminated areas or foreign material inclusions (e.g. forgotten tapes), it is essential to choose a favourable sample orientation and/or a higher resolution. For inspections of larger plates or even structure components these requirements are usually not accomplishable. Regarding the example above, it is clear that the delaminated areas were found by using CT but it is also likely that defects like that could be overseen - even more when delaminations are not expected and their location in the material is unknown. With air-coupled ultrasound and lock-in thermography techniques it is much easier to find and locate such kind of planar defects.

\subsection{Detection of Porosity in Ceramic Interfaces}

The examination of interface layers of composite structures is another important and also challenging issue for non-destructive inspection. In the following example an in-situ joined structural element of $\mathrm{C} / \mathrm{C}$-SiC material was investigated. This component was designed for the reentry test vehicle named EXPERT. It is part of a load introduction concept that is used for connecting the space capsule's ceramic nose cap structure with the metallic substructure underneath. During the design and qualification phase, a batch of demonstrators was produced in order to verify the maximum load capacity of this important CMC component by mechanical testing. These tests are described in detail in previous publications [14-16]. Figure 7 shows an air-coupled ultrasound scan of such an untested demonstrator while the geometry of the CMC component is illustrated by a semi-transparent 3-dimensional CT image view (Fig. 8).

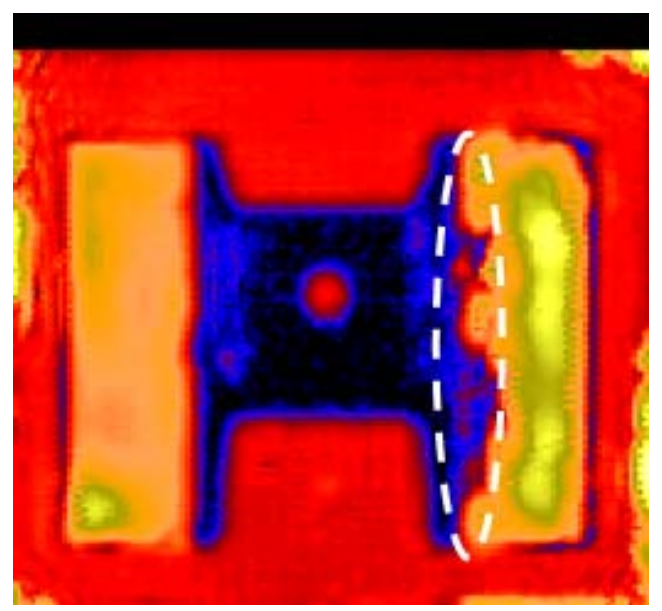

Fig. 7. Air coupled ultrasound inspection of a C/C-SiC component with increased porosity (marked region)

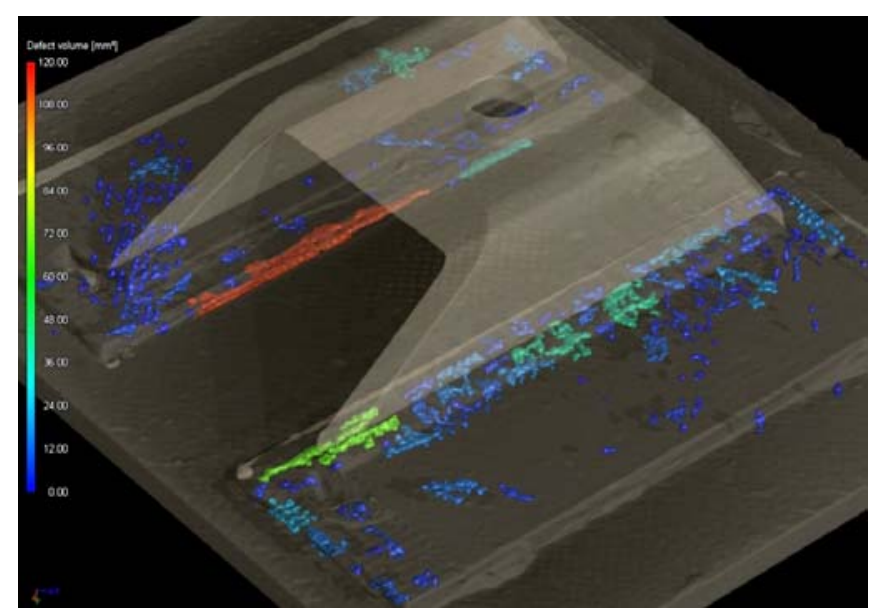

Fig. 8. Semi-transparent 3D-CT view of the C/C-SiC demonstrator with internal porosity in the joining interface 
The C/C-SiC nose cap structure was manufactured with the liquid silicon infiltration process (LSI) which was developed by DLR since the mid 1980s. In-situ joining in this context means that the joining process is an integral part of the LSI manufacturing route. The upper part and the base plate of this component are glued together with a carbon rich substance in the $\mathrm{C} / \mathrm{C}$ intermediate stage. Afterwards the assembly is undergoing the siliconization process in which the contact zone transforms into a thin SiC contact layer. Depending to the consistence and the thickness of this contact substance and the way of its application to the surfaces, it is more or less likely that there will be residual porosity in the joining interface. Before the mechanical testing, the whole batch of about $24 \mathrm{CMC}$ demonstrators was investigated by CT. It was found that some of the test samples didn't show any porosity at all while others revealed regions of significant porosity at the interfaces [16]. However, after the mechanical tests, two types of failure mode were observed: a rupture somewhere in the ceramic structure or a disintegration of the joining interfaces. A correlation between the interface porosity and the type of component failure is likely but the specific influence of porosity aggregation on the mechanical properties of the CMC component is still investigated. Figure 7 shows an air-coupled ultrasound scan of an untested demonstrator with its two rectangular shaped contact zones to the CMC base plate which - transferred to the application scenario - would represent the nose cap structure. The ultrasound transmission through the left joining area looks quite uniform which indicates no significant defects or inhomogeneous material distribution whereas the right joining area shows local attenuation of the acoustic signal (marked region). The distribution of dark blue colour in this area indicates a local increased porosity at the joining interface which is a potentially failure critical zone. A CT scan of another demonstrator of this batch also shows a certain amount of porosity at the interface of the joining layers (Fig. 8). The ability of illustrating the object in a semi-transparent way really helps to imagine at which location of the CMC component the defects are concentrated. As indicated by the scale, the defect finder module of the visualization software assigned colours for different defect volume classes. Most of the pores are quite small and the distribution is more or less widespread within the joining interface. The bigger pores, marked with green and red colour, are agglomerations of numerous small pores which are connected in a network-like structure.

All these specific details about pore size distribution, shape and agglomeration tendencies are not detectable with NDE techniques like ultrasound and thermography inspection. On the other hand lock-in thermography may provide sufficient and also valuable information about failure critical CMC components with less technical effort and measurement time especially when applied to large objects as for example a complete nose cap structure. However, the CMC demonstrators were also inspected after the mechanical test campaign. As already mentioned, two types of component failure were observed: a rupture somewhere in the CMC body or a more or less visible separation at the joining interfaces. With both, ultrasound inspection and lock-in thermography it is possible to make a distinction which kind of failure mode happened. Figure 9 shows air-coupled ultrasound scans of two different C/C-SiC demonstrators after the test. Obviously the contact zones of sample 13/1 remained intact as indicated by the uniform colour distribution whereas sample 23/1 shows massive signal attenuation in these areas. This is a hint of complete separation at the joining interface. Figure 10 shows the corresponding OLT phase images of the same two demonstrators. The inspection was performed from the side of the demonstrator's base plate which means that the thermal wave had to pass through approx. 5-6 mm C/C-SiC material. Thus, a low excitation frequency was chosen $(f=0.005 \mathrm{~Hz})$ which explains the relatively diffuse images. The results agree very well with the ultrasound scans: while sample $13 / 1$ shows an intact interface, the shaded areas of sample 23/1 indicate that there is a thermal barrier which means a loss of contact at the interface zones. This demonstrates very clearly that a simple lock-in thermography inspection from the outside of a CMC nose cap structure would be sufficient to answer the question for intact joining layers.

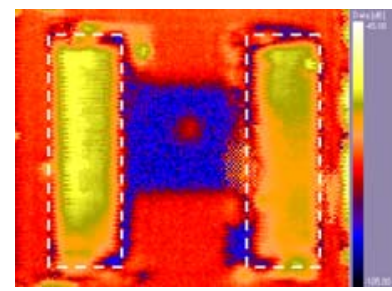

Sample \#13-1

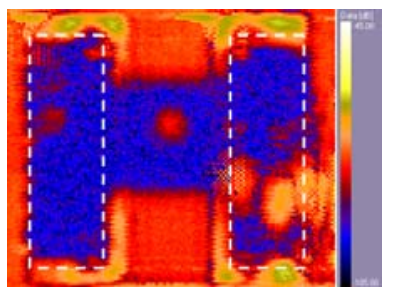

Sample \#23-1

Fig. 9. Air-coupled ultrasound inspection of different C/C-SiC components after mechanical testing

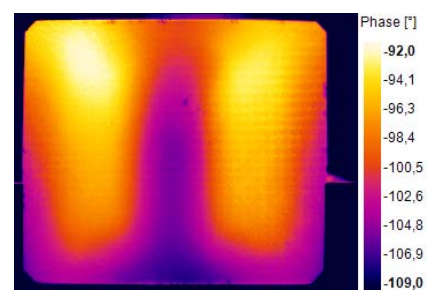

Sample \#13-1

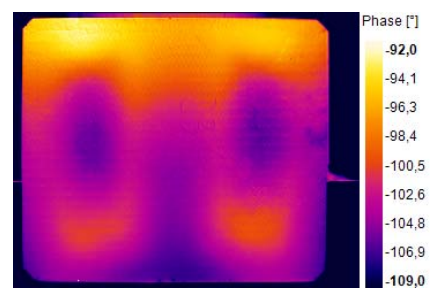

Sample \#23-1
Fig. 10. OLT inspection of different C/C-SiC components after mechanical testing $(f=0.005 \mathrm{~Hz})$

\subsection{Delamination and Porosity in Oxide CMC}

Wound highly porous oxide matrix ceramic composite material (WHIPOX) is consisting of alumina oxide fibres which are embedded in mullite- or alumina-rich matrices. Structure components made of WHIPOX material are damage tolerant and stable against oxidation. Thus, potential application fields are thermal protection systems, combustion chambers and gas turbines. The microstructure of this CMC material is a result of its specific manufacturing process. WHIPOX materials are produced by a continuously working, automated winding technique. In order to obtain flat plates, the material is then removed from the mandrel and straightened. To achieve a higher density and a better homogeneity, the green plates are finally pressed and then sintered in air at $1300^{\circ} \mathrm{C}[17,18]$. 
WHIPOX plates can be produced with different fibre roving angles and therefore with varying porosity distribution and laminate structure. For the design of structure components and the numerical modelling of this oxide $\mathrm{CMC}$ material, it is necessary to obtain reliable material properties from mechanical testing. As part of an ongoing study, batches of mechanical test samples were cut out of different WHIPOX plates. The one shown here has a fibre orientation of $\pm 45^{\circ}$ (Fig. 11-14) and dimensions of approx. $360 \times 250 \times 5 \mathrm{~mm}^{3}$ (length/width/thickness). This plate was inspected with contact less air-coupled ultrasound and lock-in thermography at different excitation frequencies $(0.3 \mathrm{~Hz}, 0.06 \mathrm{~Hz}$ and $0.01 \mathrm{~Hz}$ ). The objective of this non-destructive pre-investigation was to gain new insight about the homogeneity of the WHIPOX material in order to cut out mechanical test samples which are really comparable. In other words, areas with aggregations of material defects (e.g. porosity) had to be found. The trough transmission scan obtained by air-coupled ultrasound inspection, reveals some areas of significantly damped signal amplitude which is indicated by the dark blue colour (Fig. 11). This distribution pattern can be confirmed by OLT: the phase image of the measurement at $0.01 \mathrm{~Hz}$ (Fig. 12) shows that there are defects of similar size detectable which occur at the same locations of the CMC plate.

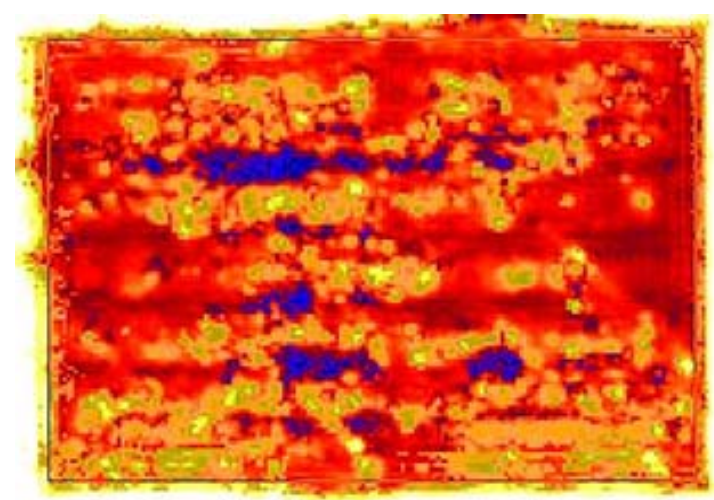

Fig. 11. Air-coupled ultrasound image of oxide CMC material with internal defects (plate \#1262)

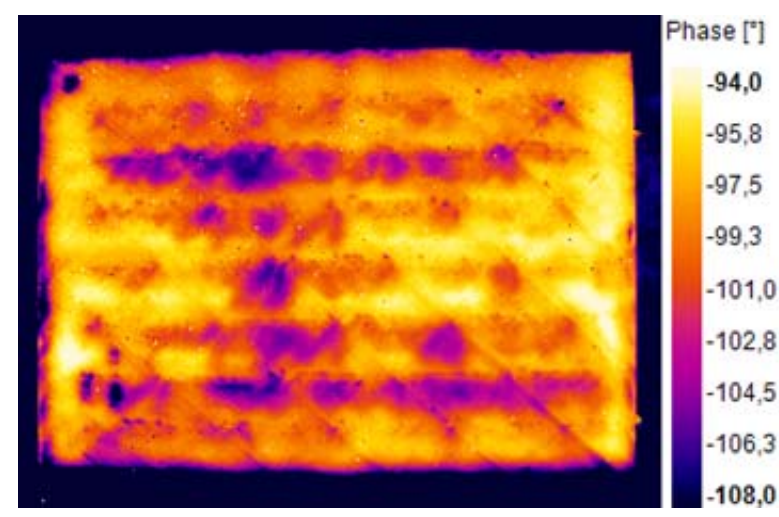

Fig. 12. OLT image of the same oxide CMC plate as shown on the left $(f=0.01 \mathrm{~Hz}$, plate \#1262)

While the trough transmission mode of ultrasound inspection does not provide any depth information, the fact that with OLT these defects are only visible at lower excitation frequencies leads to the conclusion that the defects are located a few millimetres below the surface, somewhere in the middle of the plate's thickness. Although details in phase images appear more and more diffuse due to increasing lateral heat transfer, Figure 12 shows quite well some of the crossover points of the ceramic fibre rovings and therefore the fibre orientation of $\pm 45^{\circ}$. Obviously, there is a correlation between the fibre orientation, the resulting crossover points within the fibre layup and the occurrence of local defects. However, this seems to be confirmed by the $\mu$-CT cross-sectional images below (Fig. 13).
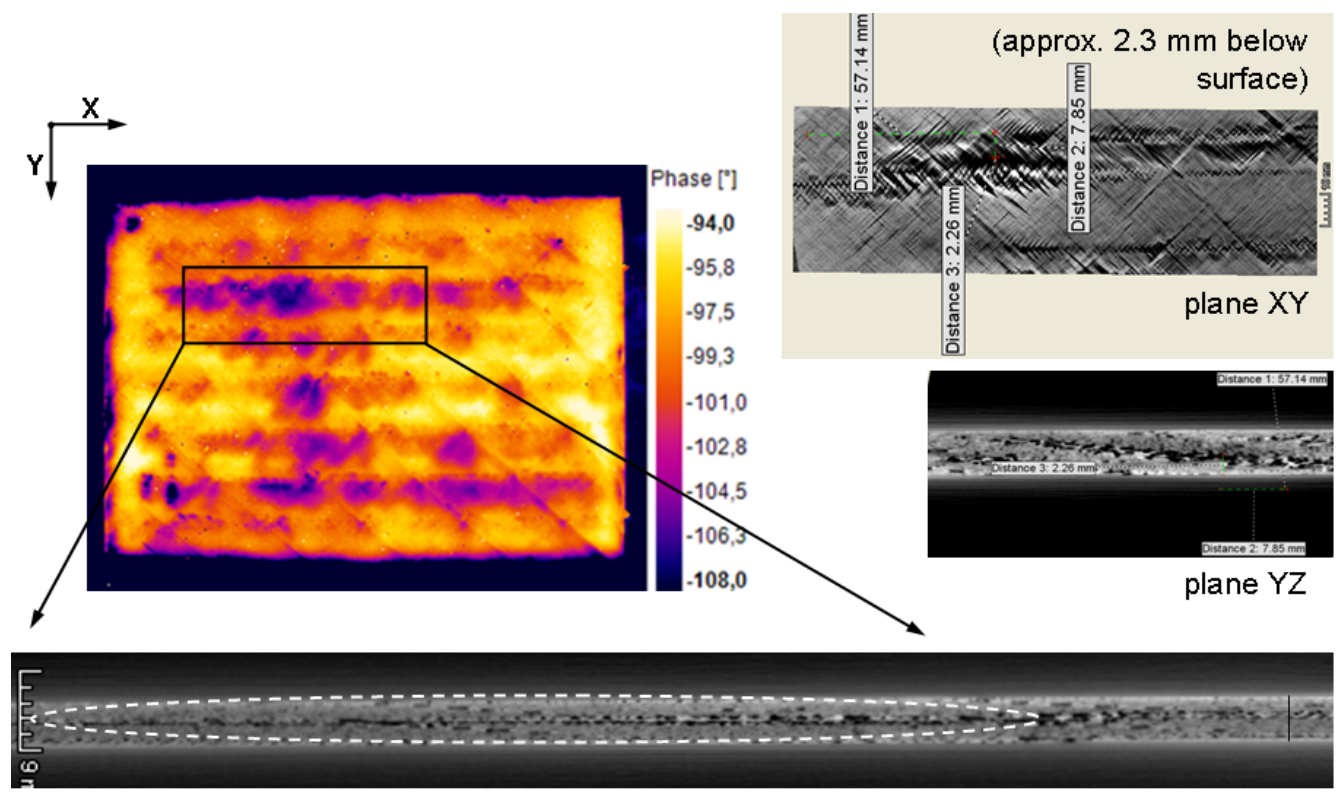

plane $X Z$

Fig. 13. Comparison of an OLT image with 2-dimensional cross sections from CT analysis 
According to the CT cross section views, there is a delaminated area which can be seen in its full elongation on plane XZ. It also confirms the assumption that the defect must be somewhere in the middle of the CMC plate. However, CT inspection confirms that it is around $2.3 \mathrm{~mm}$ below the surface. The CT inspection of Figure 13 is showing images of a so called region of interest scan (ROI), focused to one of the most noticeable defect sites in this plate. The ROI location was determined on the basis of the results of OLT and ultrasound inspection. The voxel size is approx. $71 \mu \mathrm{m}$ with a magnification factor of 2.8. Of course a local CT inspection reveals much more detailed information about the type and size of a defect and its exact location within the laminate structure. On the other hand ultrasound and OLT inspections are more suitable to get an overview of correlations between defect patterns and periodic microstructural details of a composite material. Figure 14 shows an overlay image of the OLT measurement result at $0.01 \mathrm{~Hz}$ (Fig. 12) and an excerpt of the cut out plan for the tensile test samples (a). The fibres were in $\pm 45^{\circ}$ orientation to the loading direction. The waisted geometry of the tensile test samples is shown in Figure 14b. The waisted gauge section was about $8 \times 5$ $\mathrm{mm}^{2}$ with a length of $50 \mathrm{~mm}$ (Fig. $14 \mathrm{c} \& \mathrm{~d}$ ). Figure 14c is showing the side view of sample $45 \mathrm{Z3}$ while $14 \mathrm{~d}$ shows the side view of sample 45Z6. It is obvious that in the latter one there is a defect horizon which is almost located in the middle of the sample's thickness. Most of the test samples were cut out from regions with no detectable defects (e.g. samples 45Z1, $45 Z 2$ and 45Z3) but there were also a few test samples which were prepared from a region with significantly increased porosity (e.g. sample 45Z6) in order to quantify the expected deviations in mechanical properties.

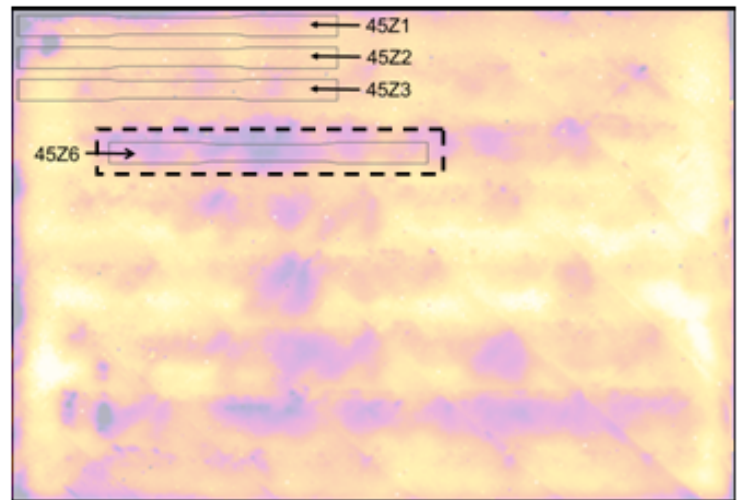

(a)

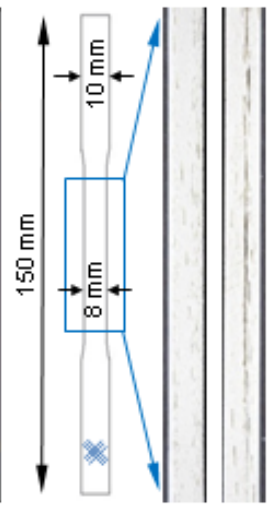

(b) (c) (d)

Fig. 14. (a) combination of OLT image and cut out plan for mechanical test samples, (b) tensile test sample geometry and fibre orientation, (c) side view of tensile test sample \#45Z3, (d) side view of tensile test sample \#45Z6

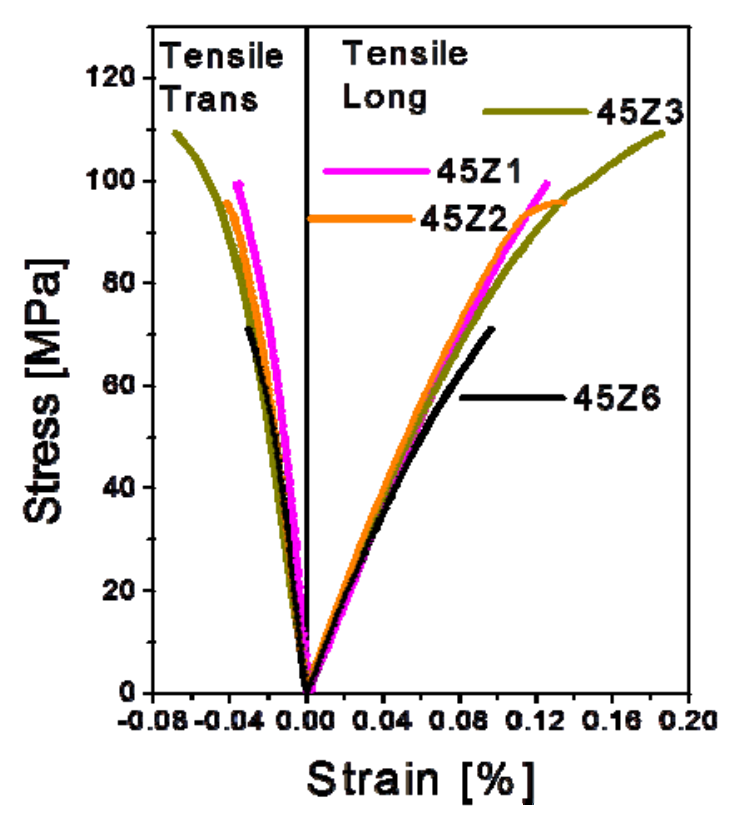

Fig. 15. Tensile testing of CMC composite with longitudinal and transverse stress-strain curves
The mechanical tests were performed at room temperature under quasi-static tensile loading. The specimens were tested in a spindle driven testing machine and the strain was measured with strain gauges in longitudinal and transverse directions.

According to the results shown in Figure 15, there is considerable different mechanical behaviour between the specimens $45 Z 6$ and $45 Z 1-Z 3$. In the case of $45 Z 6$, the material fails already at a relatively low stress level of $70 \mathrm{MPa}$ which is approx. only $70 \%$ compared to the load levels of the other specimens which were prepared from areas without detectable defects (Fig. 14a). The calculated Young's modulus of Z6 is $93 \mathrm{GPa}$ which is lower than that of the samples Z1-Z3 by a factor of 0.9 . It is obvious that there is a correlation of the porosity of WHIPOX material and the mechanical properties of test samples. 


\section{Conclusions}

Computed tomography provides more detailed information about a composite material's microstructure than any other non-destructive technique and is therefore an excellent reference method for improved signal interpretation. But focussing the smallest details also means that the detectable sample volume becomes quite small. Thus, the view turns more and more into a material characterization in which the context between structural aspects and observed defect patterns are gradually lost from sight. In terms of a consistently process monitoring and efficient quality assurance other strategies are needed. In contrast, inspection techniques like contactless air-coupled ultrasound or optically excited lockin thermography both turned out to be quite good for reliable overview measurements. The examples shown here have proven that these methods are even more suitable for inspections on larger structure components since they can be operated within a minimum of measurement time and by far less technical effort than with CT.

\section{REFERENCES}

[1] Weimer C. et al., "Process Attendant Failure Analysis (PROSA) - a Significant Component for Cost-Efficient Manufacturing and Analysis of CFRP Structures" (in German). DGLR Jahrbuch 2006, Vol. III, pp. 1633-1643, 2007.

[2] Ullmann T., Schmidt T., Hofmann S. and Jemmali R., "In-line Quality Assurance for the Manufacturing of Carbon Fiber Reinforced Aircraft Structures". Proceedings of $2^{\text {nd }}$ International Symposium on NDT in Aerospace 2010, available online: http://www.ndt.net/article/aero2010/papers/tu1a4.pdf, 2010.

[3] Ullmann T., Aoki R. and Schmidt T., "Lock-in Thermography for Process Integrated Non-Destructive Evaluation of Carbon Fibre Reinforced Aircraft Structures". Proceedings of $10^{\text {th }}$ Quantitative InfraRed Thermography conference, paper QIRT2010-140, Québec (Canada), 2010.

[4] Ibarra-Castanedo C. et al., "Active Infrared Thermography Techniques for the Non-destructive Testing of Materials". Capter XIV of the book: "Ultrasonic and Advanced Methods for Nondestructive Testing and Material Characterization", ed. Chen C.H., pp. 325-348, 2007.

[5] Chatzigeorgiou L., "Examination of Leakage Behaviour on Vacuum Infusion Processes" (in German). Diploma Thesis, University of Stuttgart (2005).

[6] Wu D., "Lockin-Thermography for Non-destructive Material Testing and Material Characterization" (in German). Doctoral thesis, University of Stuttgart (1996).

[7] Brandt R., Jaroma-Weiland G., Neuer G., Pohlmann P. and Schreiber E., "Thermal Behaviour of C/C-SiC" (in German). Working Report, SFB 259, TP B3, University of Stuttgart (1998).

[8] Kochendörfer R. and Lützenburger N., "Applications of CMCs Made via the Liquid Silicon Infiltration (LSI) Technique". In: High Temperature Ceramic Matrix Composites, ed. by Krenkel W., Naslain R. and Schneider H., Wiley-VCH, pp. 277-287, 2001.

[9] Mühlratzer A. and Leuchs M., "Applications of Non-Oxide CMCs".In: High Temperature Ceramic Matrix Composites, ed. by Krenkel W., Naslain R. and Schneider H., Wiley-VCH, pp. 288-298, 2001.

[10] Leuchs M., "Ceramic Matrix Composites - Status of Materials and Applications in CVI-Technology". Proceedings of $10^{\text {th }}$ Int. Conf. and Exhibition of the Europ. Ceram. Soc., Berlin, 2007, paper available online: http://www.ecers2007berlin.de/abstracts files/manuscript files/5/134/J\%20134\%20Martin\%20Leuchs.pdf.

[11] Hackemann S., Schmücker M., DLR Cologne, pers. communication, 2012.

[12] Gerhard H., "Development and Testing of new dynamic Speckle Techniques for Non-destructive Material and Component Testing" (in German). Doctoral thesis, University of Stuttgart (2007), available online: http://elib.uni-stuttgart.de/opus/volltexte/2007/3297/pdf/Dissertation Henry Gerhard.pdf.

[13] Stößel R., "Air-Coupled Ultrasound Inspection as a New Non-Destructuctive Testing Tool for Quality Assurance". Doctoral thesis, University of Stuttgart (2004), available online: http://elib.uni-stuttgart.de/opus/volltexte/2004/1622/pdf/Diss Stoessel.pdf.

[14] Stubicar K. and Reimer T., "Thermo-Mechanical Design of the Expert Nose and Testing of the Load Introductions". Proceedings of $6^{\text {th }}$ European Workshop on Thermal Protection Systems and Hot Structures, Stuttgart, 2009, paper available online: http://elib.dlr.de/61400/1/6-TPS-WS-stubicar-reimer.pdf

[15] Zuber C., Reimer T., Stubicar K., Heidenreich B. and Hald H., "Manufacturing of the CMC Nose Cap for the EXPERT Spacecraft". Proceedings of $34^{\text {th }}$ Int. Conf. and Exposition on Advanced Ceramics and Composites, ICACC2010, Daytona Beach/FL, USA, 2010, available online: http://elib.dlr.de/67307/1/ICACC 2010 paper.pdf

[16] Ullmann T., Jemmali R., Hofmann S., Reimer T., Zuber C., Stubicar K. and Weihs H., "Computed Tomography for Non-Destructive Inspection of Hot Structures and TPS Components". Proceedings of $6^{\text {th }}$ European Workshop on Thermal Protection Systems and Hot Structures, Stuttgart, 2009, paper available online: http://elib.dlr.de/62833/1/ESA-TPS-WS2009 Paper ULLMANN.pdf.

[17] Schmücker M., Grafmüller A. and Schneider H., "Mesostructure of WHIPOX all oxide CMCs". Composites: Part A, 34, pp. 613-622, 2003.

[18] Göring J., Hackemann S. and Kanka B., "WHIPOX ${ }^{\circledR}$ : a Fibre Reinforced Oxide Ceramic Matrix Composite for long-term High Temperature Applications" (in German). Materialwiss. u. Werkstofftech., 38, No. 9, pp. 766-772, 2007. 